\title{
Spinal Tuberculosis Evaluated by Means of 18F-FDG PET/CT: Pilot Study
}

\author{
Christa Zinn, Mariza Vorster and Mike Machaba Sathekge*
}

Department of Nuclear Medicine, University of Pretoria and Steve Biko Academic Hospital, Pretoria, South Africa

\begin{abstract}
Introduction: Spinal tuberculosis is a serious health care problem and diagnosis is often difficult because many diseases may have similar imaging findings. Hence the need to describe findings of spinal tuberculosis with 2-[fluorine 18]fluoro-2-deoxy-D-glucose (FDG) positron emission tomography (PET) when compared to CT.

Materials and Methods: Sixteen consecutive patients who underwent 18F-FDG PET/CT and known to have or subsequently were proven to have spinal tuberculosis were analyzed. All patients included underwent whole-body 18FFDG PET imaging; none of the patients were on tuberculostatics at the time of the PET investigation. We evaluated 18FFDG uptake visually and semiquantitatively using standardized uptake values (SUVmax) and the ratio of lesion to normal liver (S/L ratio), respectively. The presence of spinal and extraspinal lesions was evaluated histopathologically and by the radiologic findings noted on diagnostic CT. Diagnostic Computed Tomographic (CT) findings were reviewed and compared to $18 \mathrm{~F}-\mathrm{FDG} \mathrm{PET} / \mathrm{CT}$.

Results: Only 4 patients had one spinal lesion, while 12 (75\%) patients had 2 or more lesions. Those patients that had three or more lesions demonstrated intense FDG uptake with a median of 11.34 (3.8-20.9). Ten (62\%) of the patients had both spinal and extraspinal lesions. In most of the cases (75\%), the lumbar spine was involved as opposed to the thoracic vertebrae. 18F-FDG PET detected all 39/39 (100\%) clinically (sites of disease that resulted in symptoms or signs such as pain, swelling, neurological fallout etc.) and histologically (as reported by the laboratory) observed spinal sites. The median of the SUVmax for spinal lesions was 6.06 (ranging from 2.63 to 16.78). Diagnostic CT reported corresponding morphological changes in only 27 (69\%) of the spinal sites identified on 18F-FDG PET/CT. 18F-FDG PET/CT detected 60 of $60(100 \%)$ clinically observed extraspinal sites including pulmonary TB, nodal disease and other musculoskeletal sites versus the 43 of $60(71 \%)$ detected by diagnostic CT only. The median of the SUVmax was 3.61 for nodal sites (ranging from 1.71 to 10.79 ) and 3.38 for pulmonary sites (ranging from 1.23 to 5.93 ).

Conclusion: The results suggest that $18 \mathrm{~F}-\mathrm{FDG}$ PET/CT is more effective compared to diagnostic CT only in the identification of sites of active disease and contributes to a better evaluation of extraspinal involvement in TB patients. It also appears to be a very promising imaging modality for the identification of the most appropriate biopsy site, staging disease, predicting multi-drug resistance and monitoring response to therapy.
\end{abstract}

Keywords: FDG PET, spine, TB.

\section{INTRODUCTION}

In Human Immunodeficiency Virus/Acquired Immune Deficiency Syndrome (HIV/AIDS) and Mycobacterium tuberculosis (TB) infection, early identification and diagnosis of active disease is the key to effective control of the disease [1]. Globally, TB continues to be one of the leading infectious causes of morbidity and mortality, accounting for an estimated 9 million cases and 2 million deaths per year [1-4]. The effect of the dual HIV and TB epidemics is felt most acutely in developing countries, with recent estimates of dual infection rising rapidly. HIVinfected patients are at a 20 -fold increased risk of contracting TB when compared with HIV-non-infected peers [3, 5-7]. Therefore, there is an increasing clinical need for effective early non-invasive identification and diagnosis of TB. Early identification of possible drug resistant disease is similarly of

*Address correspondence to this author at the Department of Nuclear Medicine, University of Pretoria and Steve Biko Academic Hospital, Private Bag X169, Pretoria, 000, South Africa; Tel: +27 12354 1794;

Fax: +27 12354 1219; E-mail: mike.sathekge@up.ac.za great importance since it impacts on management. The vertebral column is the most common site of osseous involvement, comprising in most series about $50 \%$ of cases of skeletal TB [8]. The most common location is the vertebral body of $\mathrm{L} 1$. If untreated, spinal TB carries the risk of serious complications due to vertebral collapse and spinal cord compression. Diagnosis is often difficult because many diseases such as gram positive, gram negative and fungal infctions as well as malignant disease processes involving the vertebrae may lead to similar imaging findings [9-12]. In addition fluid for diagnostic analysis is often unavailable in extra-pulmonary TB including TB of the spine [13]. Currently Magnetic Resonance Imaging (MRI) and to a lesser extent Computed Tomography (CT) are routine imaging modalities for the evaluation of spinal TB. CTguided fine needle aspiration is a reasonably accurate means of obtaining material for histopathological evaluation and culture, but is unable to differentiate active disease from old changes. It also has a lower sensitivity for detection of early skeletal disease compared to 2-[fluorine 18]fluoro-2-deoxyD-glucose (18F-FDG) Positron Emission Tomography 
(PET) $[16,17]$. Spinal tuberculosis activity is mandatory for the determination of the optimal treatment strategy. As 18FFDG is known to accumulate in inflammatory cells such as neutrophils and activated macrophages at the site of inflammation $[1,14,15]$ this enables 18 F-FDG-PET/CT to be the preferred modality for real-time assessment of disease activity over CXR, CT, and MRI. Also, preliminary work shows that quantitative 18F-FDG uptake can be used to monitor the effectiveness of treatment $[1,16,17]$. Therefore, it is required to describe findings of spinal tuberculosis with 18F-FDG PET/CT when compared to diagnostic CT only.

\section{MATERIALS AND METHODS}

Approval for this study was granted by the Ethics Committee of the University of Pretoria.

Sixteen consecutive patients who underwent PET/CT and subsequently were proven to have spinal tuberculosis were analyzed. All patients were clinically diagnosed with spinal sespsis based on clinical examination and radiographs. There were 8 men and 8 women. Of the 16 patients 12 were black, 2 were caucasian and 2 were Asian. Three of the 16 patients were known to be HIV positive. The median age of the whole patient group was 36.6 years (range, 33-83 years).

Diagnosis of active tuberculosis was established by histological and culture analysis. Direct microscopy for M. tuberculosis was performed with a Ziehl-Nielsson stain (ZN). Quantification of acid-fast bacilli was performed using parameters specified by the Centers for Disease Control and Prevention (CDC). Mycobacterial cultures were performed with the MGIT system (BD Diagnostics Systems, Sparks, $\mathrm{MD})$ and subsequent sensitivities were determined using the agar proportion method by BACTEC MGIT (BD Diagnostics Systems, Sparks MD). Histological examination of biopsy samples was examined for features of mycobacterial infection including granulomata and Langerhans cells. The ZN stain was examined for acid-fast bacilli. Biopsy samples were submitted for molecular testing using the GeneXpert Diagnostics System (Cepheid, Sunnydale, CA). This platform utilizes real time PCR. The local laboratory at the study site performed microscopy and culture. PCR was performed by a private laboratory $[18,19]$.

White cell count (WCC), Erythrocyte sedimentation rate (ESR) and C-reactive protein (CRP) levels done within 7 days of the PET/CT study was recorded and correlated with the Suv max and number of sites of abnormally increased 18F-FDG accumulation.

All patients included underwent whole-body 18F-FDG $\mathrm{PET} / \mathrm{CT}$ imaging; none of the patients were on tuberculostatics at the time of the PET investigation. Following 18F-FDG PET/CT-imaging, included patients were put on TB-treatment.

A region of interest was manually drawn over a hypermetabolic area on transaxial attenuation corrected PET images to obtain the SUVmax. The SUV formula: SUV $=$ radiotracer activity $\times$ weight of the patient/injected dose, which characterises the relative concentration of the radiotracer in the lesion of interest. We used the SUVmax for the pixel with the greatest uptake in the lesion (maximum pixel SUV). All areas that demonstrated an SUVmax that exceeded the SUV average of a ROI drawn over the liver was considered to be representative of active tuberculosis.

Quantitative 18F-FDG PET/CT imaging results (averaged 18F-FDG SUVmax values derived imaging and the ratio of lesion to normal liver (S/L ratio) and the used of low dose CT for anatomical correlation), were related to diagnostic $\mathrm{CT}$.

\section{F-FDG PET/CT Imaging}

Whole body $18 \mathrm{~F}-\mathrm{FDG}$ PET/CT scans were acquired on a dedicated PET-CT scanner (Biograph, Siemens) from the skull top to the pelvis. Patients were required to be fasting for a minimum of 4 hours prior to $18 \mathrm{~F}-\mathrm{FDG}$ injection. Blood glycaemia was monitored with a portable capillary glucometer. Patients received a dose of 18 F-FDG based on their body weight using the following formula ((body weight/ 10)+1))*37 MBq. PET/CT imaging was performed $60 \mathrm{~min}$ after injection of $18 \mathrm{~F}-\mathrm{FDG}$. Acquisition duration per bed position was $3 \mathrm{~min}$; nine bed positions were acquired.

Images were acquired in a 3-dimensional mode and reconstructed with and without attenuation correction (CTbased) using OSEM (ordered subset expectation maximization) yielding axial, sagittal and coronal slices. 18F-FDG PET/CT images obtained were analyzed for the presence or absence of sites of active tuberculosis (areas demonstrating increased 18F-FDG accumulation with or without corresponding changes noted on $\mathrm{CT}$ ) by two experienced nuclear medicine specialists, blinded to the clinical data and to the results obtained by diagnostic CT imaging. Disagreements were resolved by consensus. For quantitative analysis, SUVmax values were obtained for all lesions identified by drawing manually defined regions of interest on the attenuation-corrected axial images. The number of sites of spinal involvement was counted and recorded. The number and SUV max values of all extraspinal sites such as lymph node or pulmonary lesions, were also recorded.

For diagnostic CT scanning, the following parameters were used: collimation of $24 \times 1.2 \mathrm{~mm}$; gantry rotation time of $500 \mathrm{~ms}$; tube voltage of $120 \mathrm{kV}$; effective tube current of $100 \mathrm{mAs}$ with online tube current modulation and a table feed of $18 \mathrm{~mm} /$ rotation. Contrast enhancement was achieved by intravenous administration of $100 \mathrm{ml}$ of non-ionic contrast material (Ultravist) at a rate of $2 \mathrm{ml} / \mathrm{sec}$. Matrix size was 512x512. Diagnostic contrast-enhanced CT images were analyzed in the presence or absence of sites of active or inactive tuberculosis by two experienced radiologists blinded to the clinical diagnosis and 18F-FDG PET findings. Disagreements were resolved by consensus. CT-criteria for the diagnosis of infection included early soft tissue edema, early periosteal reaction and the presence of focal erosions.

\section{Statistical Analysis}

Simple descriptive statistics (absolute numbers and percentages) were used to report patient characteristics and sites of involvement. Where data had a normal distribution, values were reported as means with standard deviations. In instances where data was not normally distributed, there were reported as median values with ranges/modes. 


\section{RESULTS}

Median age of the whole patient group was 36.6 years (range, 33-83 years). There were 8 men and 8 women. Of the 16 patients 12 were black, 2 were caucasian and 2 were Asian.

All 16 patients studied had active spinal disease, 6 of the 16 patients had active spinal and active pulmonary disease. Four of these 6 patients with both active spinal and pulmonary disease had in addition other musculoskeletal sites of active TB (Table 1). This would suggest that patients with pulmonary as well as spinal TB are likely to have more extensive disease with likely involvement of the musculoskeletal system elsewhere.

\section{Table 1. Extent and Severity of Involvement}

\begin{tabular}{|c|c|c|}
\hline Extent of Involvement (Spinal Sites) & No of Patients & SUV \\
\hline \hline 1 site & 4 & $4.25(2.92-5.07)$ \\
\hline 2 sites & 6 & $5.74(2.87-10.3)$ \\
\hline$>3$ sites & 6 & $11.34(3.8-20.9)$ \\
\hline
\end{tabular}

Only 4 patients had one spinal lesion, while $12(75 \%)$ patients had 2 or more lesions (Figs. 1, 2). Those patients that had three or more lesions demonstrated intense FDG uptake with a median of 11.34 (3.8-20.9) (Table 1). Ten $(62 \%)$ of the patients had both spinal and extraspinal lesions. In most of the cases (75\%), the lumbar spine was involved as opposed to the thoracic vertebrae. 18F-FDG PET/CT detected all 39/39 (100\%) clinically observed (resulting in pain, discomfort or neurological fallout or sites that could be detected by physical examination) and histological observed (sites with microscopic or culture evidence of TB) spinal sites. The median of the SUVmax for spinal lesions was 6.06 (ranging from 2.63 to 16.78 ). CT reported corresponding morphological changes in only $27(69 \%)$ of the spinal sites identified on 18F-FDG PET. 18F-FDG PET/CT detected 60 of $60(100 \%)$ clinically observed extraspinal sites including pulmonary $\mathrm{TB}$, nodal disease and other musculoskeletal sites versus the 43 of $60(71 \%)$ detected by CT (Table 2$)$. The median of the SUVmax was 3.61 for nodal sites (ranging from 1.71 to 10.79 ) and 3.38 for pulmonary sites (ranging from 1.23 to 5.93 ).

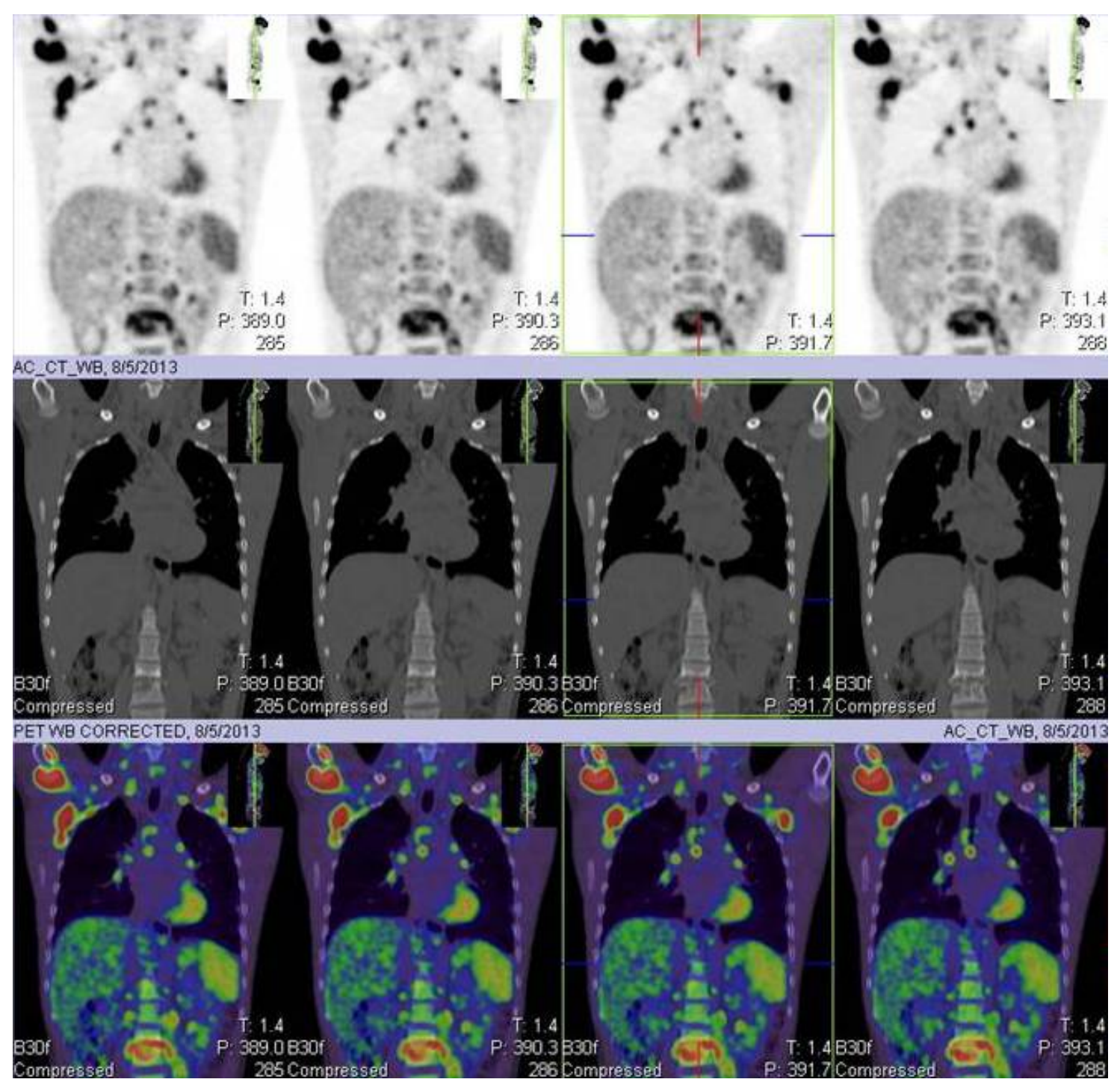

Fig. (1). 18F-FDG PET/CT demonstrating multiple spinal involvement. 


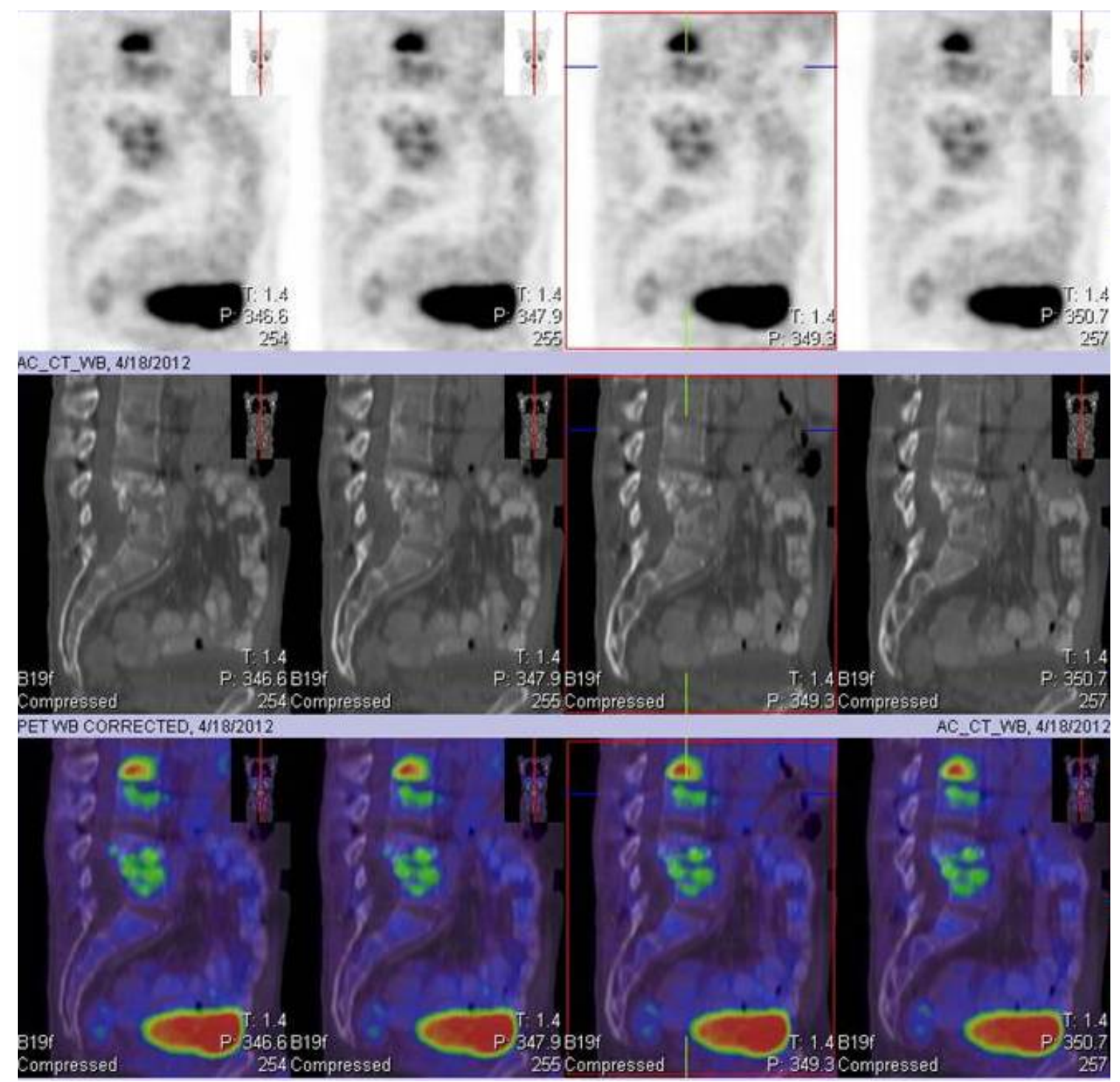

Fig. (2). A 43 year old male patient with 18F-FDG PET/CT showing multiple spinal involvement, and extraspinal involvement (nodal and splenic)

Table 2. Comparison of 18F-FDG PET and CT

\begin{tabular}{|c|c|c|}
\hline Site of Involvement & 18F-FDG PET & CT \\
\hline \hline Spinal & $39(100 \%)$ & $27(69 \%)$ \\
\hline Extraspinal & $60(100 \%)$ & $43(71 \%)$ \\
\hline
\end{tabular}

In $75 \%$ of cases active spinal disease sites involved the lumbar spine and pelvis (Fig. 3).

Patients that had both TB and HIV had in most cases the greatest number of nodal groups involved. In all patients the ESR was elevated, in most cases exceeding $50 \mathrm{~mm} / \mathrm{hr}$ (ranging from 18-122). CRP was elevated in all but one patient, ranging from 17.5-287.4 and in most cases exceeding 20. The WCC did not demonstrate any correlation with Suv max or the number of sites of active disease.

\section{DISCUSSION}

The early diagnosis and prompt treatment of spinal TB are of fundamental importance in preventing severe spinal deformity (gibbus) resulting from an acute kyphotic angulation. A delay between the initial symptoms and the etiological diagnosis is possibly caused by the low incidence of spinal $\mathrm{TB}$ and the slow development of the clinical features $[6,7]$. Time from symptom onset to diagnosis may range from several days to upto three years, but mostly spans several months $[6,7,13]$. Typically, multiple vertebral bodies and disc spaces are involved. In our study population the lumbar vertebrae were involved in $75 \%$ of cases which is consistent with what has been reported in the literature by other groups [8].

Although the ESR was abnormally elevated in all patients and CRP values were elevated in all but one patient, the absolute ESR and CRP values did not correlate with the 


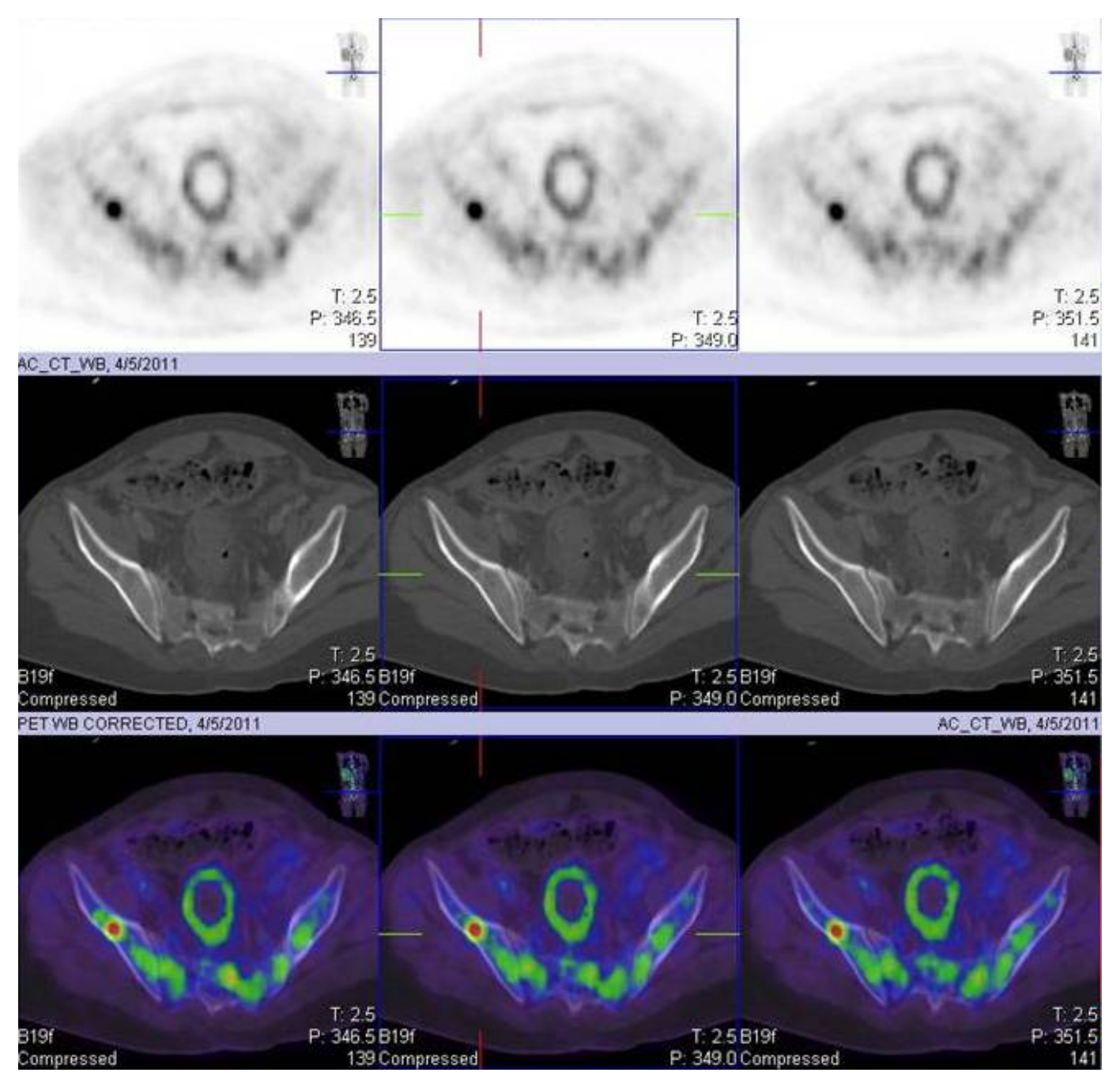

Fig. (3). 18-FDG PET/CT identifies active spinal disease and extraspinal involvement in the pelvis.

SUVmax of spinal lesions or overall disease extent in our study group. It has been reported in the literature that patients may have a normal WBC, and a normal ESR in up to $10 \%$ of the cases, which is in agreement with what we have found in our group $[4,8,18]$. Abnormal WBC, CRP level or complete blood count does not contribute to the diagnosis.

The finding of elevated ESR does not advance the diagnosis, but may be useful for follow-up of treatment response $[4,8]$. Tuberculin skin testing when positive is not of much help either especially in endemic areas or with recipients of the bacille Calmette-Guérin vaccine. Furthermore, the Mantoux and purified protein skin tests may be negative in as many as $14 \%$ of the patients with vertebral TB [18,20,21]. A negative skin test may be caused by anergy, particularly with immuno-suppressed and older patients $[18,21]$. Finally, the chest radiographs and exposure history may not be positive. Microbiological and/or histological confirmation is required for the final diagnosis of spinal TB and fine-needle aspirate, imaging-guided percutaneous biopsy, or open biopsy may be essential. Determination of spinal tuberculosis activity is mandatory for determination of the optimal treatment strategy. As 18FFDG is known to accumulate in inflammatory cells such as neutrophils and activated macrophages at the site of inflammation $[1,14,15]$ this enables 18 F-FDG-PET/CT to be the preferred modality for real-time assessment of disease activity over CXR, CT, and MRI. 18F-FDG PET/CT was superior in the identification of involved nodal basins compared to $\mathrm{CT}$ alone. In our group, the three patients that had both TB and HIV had the greatest number of nodal groups involved (an average of 4 nodal groups per HIV positive patient compared to an average of 1.6 nodal groups in HIV negative patients). Sathekge et al. has shown that in cases where 5 or more nodal basins demonstrated active disease, treatment resistance was more likely [8]. This suggests that $18 \mathrm{~F}-\mathrm{FDG}$ PET is a valuable imaging modality for the prediction of possible drug resistance thereby impacting on management $[1,8]$.

The number of involved nodal basins has been linked to an increased likelihood of drug resistant tuberculosis $[5,8]$. Four of 6 patients who had both active spinal and pulmonary disease had in addition other musculoskeletal sites of active tuberculosis. This would suggest that patients with pulmonary as well as spinal $\mathrm{TB}$ are likely to have more extensive disease and may benefit from investigation for involvement of the musculoskeletal system elsewhere. 
Although no imaging modality can confidently differentiate benign from malignant disease 18F-FDG $\mathrm{PET} / \mathrm{CT}$ has proven to be a valuable modality for the identification of the most appropriate biopsy site for histopathological evaluation and diagnosis $[16,17,22]$. Secondly even though definitive diagnosis and exclusion of active TB infection by culture and examination of tissue specimens are always recommended, 18F-FDG-PET/CT may contribute to earlier detection/diagnosis [1,23-25].

This current study suggests that $18 \mathrm{~F}-\mathrm{FDG}-\mathrm{PET} / \mathrm{CT}$ may be a useful tool in the assessment of the extent of active disease involvement prior to treatment and potentially for the assessment of treatment response [1]. This is in agreement with work done by Schmitz et al. [8]. Preliminary work done on animal models as well as humans has demonstrated that quantitative $18 \mathrm{~F}-\mathrm{FDG}$ uptake can be used to monitor the effectiveness of treatment $[1,16,17]$. Furthermore, this pilot study shows that extensive involvement of 3 or more vertebral sites appears to have more intense $18 \mathrm{~F}-\mathrm{FDG}$ uptake $=11.34(3.8-20.9)$. This finding needs to be evaluated further for possible prognostication and to determine the duration and type of therapy.

\section{CONCLUSION}

The results suggest that $18 \mathrm{~F}-\mathrm{FDG}$ PET/CT is more efficient in the identification of sites of active disease and contributes to a better overall staging of extraspinal involvement in TB patients. It also appears to be a very promising imaging modality for the identification of the most appropriate biopsy site, evaluating disease extent, predicting multi-drug resistance and monitoring response to therapy.

\section{CONFLICT OF INTEREST}

The authors confirm that this article content has no conflicts of interest.

\section{ACKNOWLEDGEMENTS}

Declared none.

\section{REFERENCES}

[1] Sathekge M, Maes A, Van de Wiele C. DG PET Imaging in HIV Infection and Tuberculosis. Semin Nucl Med 2013; 43: 349-66.

[2] WHO. Global Tuberculosis Control 2011. Geneva, Switzerland: World Health Organization; 2011. http://WWW.who.int/tb/publica tions/glob- al report/2011/gtbr11 full.pdf

[3] Kelleher H, Zaunders J. Decimated or missing in action: CD4+ T cells as targets and effectors in the pathogenesis of primary HIV infection. Curr HIV/AIDS Rep 2006; 3: 512.
[4] Sameul R, Bettiker R, Suh B. AIDS related opportunistic infections, going but not gone. Arch Pharm Res 2002; 25: 215-28.

[5] Sathekge M, Maes A, Kgomo M, et al. Use of 18-FDG PET to Predict Response to Fist-line Tuberculostatics in HIV-associated Tuberculosis. J Nucl Med 2011; 52: 880-5.

[6] Arccniegas W, Orjuela D. Extrapulmonary tuberculosis: A review of 102 cases in Pereira, Colombia. Biomedica 2006; 26: 71-80.

[7] Rivas-Garcia A, Sarria-Estrade S, Torrents-Odin C, et al. Imaging findings of Pott's disease. Eur Spine J 2012; 22: S567-78.

[8] Sathekge M, Maes A, Kgomo M, et al. Impact of FDG PET imaging on the management of TB treatment: a pilot study. Nuklear Medizine 2010; 49: 35-40.

[9] Lucio E, Adesokan A, Hadjipavlou AG, et al. Pyogenic spodilodiskitis: a radiological/ pathological and culture correlation study. Arch Pathol Lab Med. 2000; 124: 712-6.

[10] Weinberg J, Silber JS. Infections of the spine: what the orthopedis needs to know. Am J Orthop 2004; 33: 13-7.

[11] Smith AS, Blaser SI. Infectious and inflammatory processes of the spine. Radiol Clin North Am 1991; 29: 809-27.

[12] Merrick MV, Beales JS, Garvie N, Leonard RC. Evaluation of skeletal metastases. Br J Radiol 1992; 65: 803-6.

[13] Golden MP, Vikram HR. Extrapulmonary tuberculosis: an overview. Am Fam Physician 2005; 72: 1761-8

[14] Kosterink JGW. Positron emission tomography in the diagnosis and treatment management of tuberculosis. Curr Pharm Des 2011; 17: $2875-80$.

[15] Zhuang H, Alavi A: 18-Fluorodeoxyglucose positron emission tomographic imaging in the detection and monitoring of infection and inflammation. Semin Nucl Med 2002; 32: 47-59.

[16] Schmitz A, Risse JH, Grunwald F, et al. Fluorine-18 fluorodeoxyglucose positron emission tomography findings in spondylodiscitis: preliminary results. Eur Spine J 2001; 10: 534-9.

[17] Kim SJ, Kim IJ, Suh KT, et al. Prediction of residual disease of spine infection using F-18 FDG PET/CT. Spine 2009; 34: 2424-30.

[18] Winn W, Allen S, Janda W, et al. Koneman's color atlas and textbook of diagnostic microbiology, 6th ed. Philidelphia: Lippencot Williams and Wilkins; 2006.

[19] Boehme C, Nicol M, Nabeta P, et al. Feasibility, diagnostic accuracy and effectiveness of decentralized use of Xpert MTB/RIF test for diagnosis of tuberculosis and multi-drug resistance: a multicenter implementation study. Lancet 2011; 377(9776): 1495505.

[20] Long R. Smear negative pulmonary tuberculosis in industrialized countries. Chest 2001; 120: 330-4.

[21] Van Deun A, Martin A, Palomino JC. Diagnosis of drug-resistant tuberculosis: reliability and rapidity of detection. Int J Tuberc Lung Dis 2010; 14: 131-40.

[22] Ahmad S, Mokaddas E. Recent advances in the diagnosis and treatment of multidrug-resistant tuberculosis. Respir Med 2009; 103: 1777-90.

[23] Aggarwal A, Saluke P, Shekhar BR, et al. The role of Magnetic Resonance Imaging and Positron Emission Tomography combined in differentiating benign from malignant lesions contributing to vertebral compression fractures. Surg Neurol Int 2013; 4: 323-6.

[24] Goo JM, Im JG, Do KH, et al. Pulmonary Tuberculoma Evaluated by Means of FDG PET: Findings in 10 cases. Radiology 2000; 216 : 117-2.

[25] Glaudermans AWJM, Signore A. FDG-PET/CT in infections: the imaging method of choice? Eur J Nucl Med Mol Imaging 2010; 371: $986-91$ 